\title{
EDITORIALS
}

\section{Rethinking "Abnormal” Blood Pressure: What Is the Value?}

\author{
Gerald W. Smetana, MD \\ Division of General Medicine and Primary Care, Beth Israel Deaconess Medical Center, Harvard Medical School, Boston, MA, USA.
}

$\mathrm{J}$ Gen Intern Med 26(7):678-80

DOI: $10.1007 / \mathrm{s} 11606-011-1737-2$

(c) Society of General Internal Medicine 2011

$\mathrm{H}$ ypertension is a major public health priority that accounts for more adult primary care office visits than any other chronic medical condition ${ }^{1}$. According to the most recent National Health and Nutrition Examination Surveys (NHANES) data from 2005-2008, 31\% of all US adults are hypertensive based on a definition of blood pressure $\geq 140$ / $90 \mathrm{mmHg}$ or taking antihypertensive medication; ${ }^{2}$ the prevalence among African-Americans is 39\%. Using the same definition, the majority of older individuals are hypertensive: prevalence among those $\geq 65$ years of age rises to $70 \%$. Despite widespread public awareness that hypertension is a major risk factor for cardiovascular morbidity and mortality, only $81 \%$ of hypertensive adults are aware of their diagnosis ${ }^{3}, 73 \%$ are taking anti-hypertensive medication, and 50\% of patients are taking antihypertensive medication and have achieved a goal blood pressure of $<140 / 90{ }^{4}$. Even a small change in criteria for the diagnosis of hypertension would have a substantial impact on disease prevalence, labeling, burden of treatment and health care costs.

The most recent version of the Joint National Committee on Prevention, Detection, Evaluation and Treatment of High Blood Pressure (JNC-7), published in 2003, classified hypertension as a systolic blood pressure of $\geq 140 \mathrm{mmHg}$ or a diastolic blood pressure of $\geq 90 \mathrm{mmHg}^{5-6}$. The committee used lower thresholds $(\geq 130 / 80)$ for patients with diabetes or chronic kidney disease. In the general population, the committee classified blood pressures of 120-139/80-89 as pre-hypertension. New in the 2003 JNC guidelines was the focus on systolic blood pressure as the primary marker of risk, particularly among patients $>50$ years old. Most clinicians have embraced these classifications in practice.

In this issue of the Journal, Taylor and colleagues use a novel methodology to reconsider the contribution of various levels of systolic and diastolic blood pressure to overall mortality in older and younger individuals ${ }^{7}$. The authors also aimed to determine the impact of revised definitions of normal blood pressure on the prevalence of hypertension in the US. The authors made the reasonable assumption that currently available population data that stratify mortality by blood pressure values would be confounded by the impact of antihypertensive therapy. They therefore chose to examine a data set of patients $(n=13,792)$ from the NHANES I survey of 1971-1976, a time frame during which antihypertensive

Published online May 10, 2011 therapy was less commonly prescribed. All patients had complete vital status data at study entry and epidemiologic follow-up data through 1992. The primary outcome was allcause mortality. Strengths of the study include complete vital status data for the period of the study and the ability to adjust for important confounders including age, sex, smoking status, BMI, total cholesterol, income and race. In order to estimate the distribution of blood pressure values in a population of untreated individuals, the authors sought data from an era before routine treatment of hypertension; they used data $(n=6,672)$ from the National Health Examination Survey (NHES) from 1959 through 1962.

For individuals $>50$ years of age, a $\mathrm{J}$-shaped relationship existed between diastolic blood pressure at study entry and mortality a mean of 18 years later. Mortality was lowest for a diastolic blood pressure of $80-89 \mathrm{mmHg}$; however, this relationship largely disappeared after adjusting for systolic blood pressure. In contrast, the J-shaped relationship between systolic blood pressure and mortality, with a nadir at 110$119 \mathrm{mmHg}$, was unaffected by adjustment for diastolic blood pressure. The opposite relationship was apparent when studying patients who were $\leq 50$ years of age at study entry. There was a modest association between mortality and systolic blood pressure that disappeared after adjusting for diastolic blood pressure (other than for those with baseline values of $\geq 200 \mathrm{mmHg}$, a finding present in $<1 \%$ of younger study subjects). In contrast, mortality rates increased among subjects with diastolic blood pressures of $\geq 100$; this finding persisted after adjustment for systolic blood pressure. The observation that the importance of diastolic and systolic blood pressure differs by age is consistent with the findings of JNC-7 ${ }^{5}$

The authors applied these results to the blood pressure distribution from the NHES cohort of individuals from the 1959 population data. They concluded that risk was unambiguously increased for younger individuals only if systolic blood pressure was $\geq 200 \mathrm{mmHg}$ or diastolic blood pressure $\geq 100 \mathrm{mmHg}$, and for older individuals only if systolic blood pressure was $\geq 140 \mathrm{mmHg}$. After applying these criteria, the number of adult Americans in 2008 with a normal blood pressure increases from 62 million (28\%) to 163 million (74\%). The number of US adults with the label of abnormal blood pressure would fall by over $60 \%$.

Hypertension is costly to treat. When considering direct as well as downstream costs associated with excess cardiovascular disease, projected expenditures in the US were 69.9 billion USD in 2010; this is expected to nearly double in the next 10 years ${ }^{8}$. If even a modest proportion of these dollars are spent unnecessarily because of over labeling, this would have significant policy implications. In a time of an inadequate primary care workforce to meet current and anticipated future demand, fewer "unnecessary" office visits would occur using 
the new diagnostic scheme. Fewer individuals would be subject to a "labeling" effect whereby they consider themselves to be patients or chronically ill. Reduced labeling would have implications for insurability, particularly in regard to disability, life and long-term care insurance. Antihypertensive therapy itself is costly and carries the potential for drug-related adverse effects; these would diminish under this new diagnostic scheme.

However, several important caveats exist when assessing the findings of Taylor and colleagues. First is the decision to use all-cause mortality as the primary outcome. The authors selected this outcome as it was readily available and was an unambiguous outcome. This may underestimate the value of antihypertensive therapy. Nonfatal stroke and myocardial infarction are of great importance to patients and a source of substantial morbidity; the use of all-cause mortality as the outcome does not capture these events. In addition, a mean of 18 years of follow-up does not capture all meaningful benefits of antihypertensive therapy. In particular, in the cohort of individuals less than 50 years old, decades of follow-up would be required to observe the full expected reduction in cardiovascular mortality.

The authors selected the NHANES I cohort in order to identify patients who were unlikely to be exposed to antihypertensive therapy. However, a non-trivial number of these patients actually received antihypertensive therapy. In the NHANES I study, 37\% of people with hypertension (defined at that time as blood pressure $\geq 160 / 95$ ) were receiving treatment 9. Data from NHANES III reveal that by 1991-1994, 52\% percent of hypertensive patients ( $>140 / 90$ or on antihypertensive therapy) were treated (albeit only $23 \%$ were controlled). So the observational study by Taylor and colleagues of the mortality associated with untreated hypertension actually contained a substantial number of patients who were treated during the 18-year period of study. There was an important secular trend towards increased rates of hypertension treatment over this time frame. The potential effect of this confounder is to reduce long-term mortality for any given blood pressure value at study entry because of active treatment in the observational cohort.

Taylor and colleagues define all individuals who are not normal to be abnormal. However, according to Taylor's definition, "abnormal" would include patients with pre-hypertension (according to JNC-7), a group that is not "normal" but for whom no treatment is currently recommended. An alternative and reasonable definition of abnormal according to current guidelines would be $>140 / 90$. Therefore, their categorization of unambiguously increased risk (abnormal) in older individuals occurring with a systolic blood pressure $\geq 140 \mathrm{mmHg}$ is actually consistent with current guidelines and does not differ from current terminology. Only in younger individuals does their cut point of unambiguously increased risk differ from current JNC-7 recommendations. Therefore, the impact of their newly proposed definitions would be restricted to younger individuals. Using the alternative definition of abnormal as a blood pressure $>140 / 90,61 \%$ of individuals are "normal" according to present terminology. The change in labeling as a result of the data of Taylor and colleagues would be substantially smaller than described in the paper (an increase in the percent of normal individuals from $61 \%$ to $79 \%$ ).

How do the current results differ from the existing body of literature on the contribution of various blood pressure levels on morbidity or mortality? One must learn from older data reported in the era before routine treatment of hypertension. In a meta-analysis of patient-level data from nearly 1 million people across 61 studies, the risk of death due to stroke or myocardial infarction increased approximately two fold for each $20 \mathrm{mmHg}$ increase in systolic blood pressure above $115 \mathrm{mmHg}$ or $10 \mathrm{mmHg}$ increase in diastolic blood pressure above $75 \mathrm{mmHg}{ }^{10}$. This effect was seen in individuals as young as 40 years old. In an early review of predominantly male subjects aged 25-70, the relationship between untreated diastolic blood pressure and stroke or myocardial infarction was continuous to levels as low as $76 \mathrm{mmHg}$ over an average of 10 years of follow-up ${ }^{11}$. The story is more complicated when one considers what the target blood pressure should be for individuals with established hypertension. The present study is not intended to address this question.

JNC-8 has been delayed several times; the estimated publication date is now in the spring of 2012. While advances in our knowledge of the impact of various antihypertensive medication classes on cardiovascular risk will certainly be a major aspect of this updated report, thresholds for the diagnosis of hypertension will also be an important component. The study by Taylor and colleagues will contribute to this dialogue. This study, based on a single database, and with important limitations, is not sufficiently robust to change public policy or definitions of normal blood pressure. However, this is a provocative study that asks an interesting question and yields an unexpected result. Future research, using other databases of untreated individuals, would provide additional insight into this question of great importance for the public, payers and clinicians. What exactly is abnormal?

\section{Conflicts of Interest: None disclosed.}

Corresponding Author: Gerald W. Smetana, MD; Division of General Medicine and Primary Care, Beth Israel Deaconess Medical Center, Harvard Medical School, Shapiro 621D, 330 Brookline Ave., Boston, MA 02215, USA (e-mail: gsmetana@bidmc.harvard.edu).

\section{REFERENCES}

1. Hsiao CJ CD, Beatty PC, Rechtsteiner EA. National Ambulatory Medical Care Survey: 2007 Summary. National Health Statistics Report no. 27. Hyattsville, MD: National Center for Health Statistics 2010

2. Center for Disease Control and Prevention. Vital Signs: Prevalence, Treatment, and Control of Hypertension-United States, 1999-2002 and 2005-2008. MMWR Morbidity and Mortality Weekly Report 2011;60:103-8.

3. Ostchega Y, Yoon S, Hughes J, Louis T. Hypertension awareness, treatment, and control-continued disparities in adults: United States, 2005-2006. NCHS data brief. In. Hyattsville, MD: National Center for Health Statistics; 2008.

4. Egan BM, Zhao Y, Axon RN. US trends in prevalence, awareness, treatment, and control of hypertension, 1988-2008. JAMA 2010;303:2043-50.

5. Chobanian AV, Bakris GL, Black HR, et al. The Seventh Report of the Joint National Committee on Prevention, Detection, Evaluation, and Treatment of High Blood Pressure: the JNC 7 report. JAMA 2003;289:2560-72.

6. Chobanian AV, Bakris GL, Black HR, et al. Seventh report of the Joint National Committee on Prevention, Detection, Evaluation, and Treatment of High Blood Pressure. Hypertension 2003;42:1206-52.

7. Taylor B, Wilt T, Welch H. Impact of diastolic and systolic blood pressure on mortality: implications for the definition of "normal". JGIM 2011; doi:10.1007/s11606-011-1660-6. 
8. Heidenreich PA, Trogdon JG, Khavjou OA, et al. Forecasting the future of cardiovascular disease in the United States: a policy statement from the American Heart Association. Circulation 2011;123:933-44.

9. Burt VL, Cutler JA, Higgins M, et al. Trends in the prevalence, awareness, treatment, and control of hypertension in the adult US population. Data from the Health Examination Surveys, 1960 to 1991 Hypertension 1995;26:60-9.
10. Lewington S, Clarke R, Qizilbash N, Peto R, Collins R. Age-specific relevance of usual blood pressure to vascular mortality: a meta-analysis of individual data for one million adults in 61 prospective studies. Lancet 2002;360:1903-13.

11. MacMahon S, Peto R, Cutler J, et al. Blood pressure, stroke, and coronary heart disease. Part 1, Prolonged differences in blood pressure: prospective observational studies corrected for the regression dilution bias. Lancet 1990;335:765-74. 